\title{
STRONG ASYMPTOTIC INDEPENDENCE ON WIENER CHAOS
}

\author{
IVAN NOURDIN, DAVID NUALART, AND GIOVANNI PECCATI
}

(Communicated by Mark M. Meerschaert)

\begin{abstract}
Let $F_{n}=\left(F_{1, n}, \ldots, F_{d, n}\right), n \geqslant 1$, be a sequence of random vectors such that, for every $j=1, \ldots, d$, the random variable $F_{j, n}$ belongs to a fixed Wiener chaos of a Gaussian field. We show that, as $n \rightarrow \infty$, the components of $F_{n}$ are asymptotically independent if and only if $\operatorname{Cov}\left(F_{i, n}^{2}, F_{j, n}^{2}\right) \rightarrow 0$ for every $i \neq j$. Our findings are based on a novel inequality for vectors of multiple Wiener-Itô integrals, and represent a substantial refining of criteria for asymptotic independence in the sense of moments recently established by Nourdin and Rosiński (2014).
\end{abstract}

\section{INTRODUCTION}

1.1. Overview. Let $X=\{X(h): h \in \mathfrak{H}\}$ be an isonormal Gaussian process over some real separable Hilbert space $\mathfrak{H}$ (see Section 1.2 and Section 2 for relevant definitions), and let $F_{n}=\left(F_{1, n}, \ldots, F_{d, n}\right), n \geqslant 1$, be a sequence of random vectors such that, for every $j=1, \ldots, d$, the random variable $F_{j, n}$ belongs to the $q_{j}$ th Wiener chaos of $X$ (the order $q_{j} \geqslant 1$ of the chaos being independent of $n$ ). The following result, proved by Nourdin and Rosiński in [9. Corollary 3.6], provides a useful criterion for the asymptotic independence of the components of $F_{n}$.

Theorem 1.1 (See [9]). Assume that, as $n \rightarrow \infty$ and for every $i \neq j$,

$$
\operatorname{Cov}\left(F_{i, n}^{2}, F_{j, n}^{2}\right) \rightarrow 0 \quad \text { and } \quad F_{j, n} \stackrel{\text { law }}{\rightarrow} U_{j},
$$

where each $U_{j}$ is a moment-determinat $\Theta^{*}$ random variable. Then,

$$
F_{n} \stackrel{\text { law }}{\longrightarrow}\left(U_{1}, \ldots, U_{d}\right),
$$

where the $U_{j}$ 's are assumed to be mutually stochastically independent.

In other words, Theorem 1.1 allows one to deduce joint convergence from the componentwise convergence of the elements of $F_{n}$, provided the limit law of each sequence $\left\{F_{j, n}\right\}$ is moment-determinate and the covariances between the squares

Received by the editors January 8, 2014 and, in revised form, January 12, 2015.

2010 Mathematics Subject Classification. Primary 60F05, 60H07, 60G15.

Key words and phrases. Gaussian fields, independence, limit theorems, Malliavin calculus, Wiener chaos.

The first author was partially supported by the ANR Grant ANR-10-BLAN-0121.

The second author was partially supported by the NSF grant DMS1208625.

The third author was partially supported by the grant F1R-MTH-PUL-12PAMP (PAMPAS), from Luxembourg University.

* Recall that a random variable $U$ with moments of all orders is said to be moment-determinate if $E\left[X^{n}\right]=E\left[U^{n}\right]$ for every $n=1,2, \ldots$ implies that $X$ and $U$ have the same distribution. 
of the distinct components of $F_{n}$ vanish asymptotically. This result and its generalisations have already led to some important applications, notably in connection with time-series analysis and with the asymptotic theory of homogeneous sums see [1,2, 9]. The aim of this paper is to study the following important question, which was left open in the reference 9 :

Question A. In the statement of Theorem 1.1. is it possible to remove the momentdeterminacy assumption for the random variables $U_{1}, \ldots, U_{d}$ ?

Question A is indeed very natural. For instance, it is a well-known fact (see [14, Section 3]) that non-zero random variables living inside a fixed Wiener chaos of order $q \geqslant 3$ are not necessarily moment-determinate, so that Theorem 1.1 cannot be applied in several contexts where the limit random variables $U_{j}$ have a chaotic nature. Until now, such a shortcoming has remarkably restricted the applicability of Theorem 1.1 - see for instance the discussion contained in [1, Section 3].

In what follows, we shall derive several new probabilistic estimates (stated in Section 1.3 below) for chaotic random variables, leading to a general positive answer to Question A. As opposed to the techniques applied in [9], our proof does not make use of combinatorial arguments. Instead, we shall heavily rely on the use of Malliavin calculus and Meyer inequalities (see the forthcoming formula (2.11)). This new approach will yield several quantitative extensions of Theorem 1.1, each having its own interest. Note that, in particular, our main results immediately confirm Conjecture 3.7 in [1, which means that the convergence result proved in Theorem 3.6 of [1] holds for long range dependent functionals of arbitrary Hermite rank.

The content of the present paper represents a further contribution to a recent and very active direction of research, revolving around the application of Malliavintype techniques for deriving probabilistic approximations and limit theorems, with special emphasis on normal approximation results (see [11,13, for two seminal contributions to the field, as well as $[8$ for recent developments). The reader is referred to the book [7] and the survey [4] for an overview of this area of research. One can also consult the constantly updated webpage [5] for literally hundreds of results related to the findings contained in [11,13] and their many ramifications.

1.2. Some basic definitions and notation. We refer the reader to [7, 10, for any unexplained definition or result.

Let $\mathfrak{H}$ be a real separable infinite-dimensional Hilbert space. For any integer $q \geqslant 1$, let $\mathfrak{H}^{\otimes q}$ be the $q$ th tensor product of $\mathfrak{H}$. Also, we denote by $\mathfrak{H}^{\odot q}$ the $q$ th symmetric tensor product. From now on, the symbol $X=\{X(h): h \in \mathfrak{H}\}$ will indicate an isonormal Gaussian process on $\mathfrak{H}$, defined on some probability space $(\Omega, \mathcal{F}, P)$. In particular, $X$ is a centered Gaussian family with covariance given by $E[X(h) X(g)]=\langle h, g\rangle_{\mathfrak{H}}$. We will also assume that $\mathcal{F}$ is generated by $X$.

For every integer $q \geqslant 1$, we let $\mathcal{H}_{q}$ be the $q$ th Wiener chaos of $X$, that is, $\mathcal{H}_{q}$ is the closed linear subspace of $L^{2}(\Omega)$ generated by the class $\left\{H_{q}(X(h)): h \in\right.$ $\left.\mathfrak{H},\|h\|_{\mathfrak{H}}=1\right\}$, where $H_{q}$ is the $q$ th Hermite polynomial defined by

$$
H_{q}(x)=\frac{(-1)^{q}}{q !} e^{x^{2} / 2} \frac{d^{q}}{d x^{q}}\left(e^{-x^{2} / 2}\right) .
$$

We denote by $\mathcal{H}_{0}$ the space of constant random variables. For any $q \geqslant 1$, the mapping $I_{q}\left(h^{\otimes q}\right)=q ! H_{q}(X(h))$ can be extended a linear isometry between $\mathfrak{H}^{\odot q}$ 
(equipped with the modified norm $\sqrt{q !}\|\cdot\|_{\mathfrak{H}^{\otimes q}}$ ) and $\mathcal{H}_{q}$ (equipped with the $L^{2}(\Omega$ ) norm). For $q=0$, by convention $\mathcal{H}_{0}=\mathbb{R}$, and $I_{0}$ is the identity map.

The space $L^{2}(\Omega)$ can be decomposed into the infinite orthogonal sum of the spaces $\mathcal{H}_{q}$. This is the Wiener chaos expansion, which means that any squareintegrable random variable $F \in L^{2}(\Omega)$ admits the following chaotic expansion:

$$
F=\sum_{q=0}^{\infty} I_{q}\left(f_{q}\right)
$$

where $f_{0}=E[F]$, and the $f_{q} \in \mathfrak{H}^{\odot q}, q \geqslant 1$, are uniquely determined by $F$. For every $q \geqslant 0$, we denote by $J_{q}$ the orthogonal projection operator on the $q$ th Wiener chaos. In particular, if $F \in L^{2}(\Omega)$ is as in (1.2), then $J_{q} F=I_{q}\left(f_{q}\right)$ for every $q \geqslant 0$.

1.3. Main results. The main achievement of the present paper is the explicit estimate (1.3), appearing in the forthcoming Theorem 1.2. Note that, in order to obtain more readable formulae, we only consider multiple integrals with unit variance: one can deduce bounds in the general case by a standard rescaling procedure.

Remark on notation. Fix integers $m, q \geqslant 1$. We denote by $\mathcal{C}_{q}^{\infty}\left(\mathbb{R}^{m}\right)$ the space of infinitely differentiable functions $\varphi: \mathbb{R}^{m} \rightarrow \mathbb{R}$, which are bounded together with their partial derivatives up to the order $q$. Given a function $\varphi \in \mathcal{C}_{q}^{\infty}\left(\mathbb{R}^{m}\right)$, we shall use the notation

$$
\|\varphi\|_{q}:=\|\varphi\|_{\infty}+\sum\left\|\frac{\partial^{k} \varphi}{\partial x_{i_{1}}^{k_{1}} \cdots \partial x_{i_{p}}^{k_{p}}}\right\|_{\infty},
$$

where the sum runs over all $p=1, \ldots, m$, all $\left\{i_{1}, \ldots, i_{p}\right\} \subset\{1, \ldots, m\}$, and all multi-indices $\left(k_{1}, \ldots, k_{p}\right) \in\{1,2, \ldots\}^{p}$ verifying $k_{1}+\cdots+k_{p}:=k \leqslant q$.

Theorem 1.2. Let $d \geqslant 2$ and let $q_{1} \geqslant q_{2} \geqslant \cdots \geqslant q_{d} \geqslant 1$ be fixed integers. There exists a constant $c$, uniquely depending on $d$ and $\left(q_{1}, \ldots, q_{d}\right)$, verifying the following bound for any d-dimensional vector:

$$
F=\left(F_{1}, \ldots, F_{d}\right),
$$

such that $F_{j}=I_{q_{j}}\left(f_{j}\right), f_{j} \in \mathfrak{H}^{\odot q_{j}}(j=1, \ldots, d)$ and $E\left[F_{j}^{2}\right]=1$ for $j=1, \ldots, d-1$, and for any collection of smooth test functions $\psi_{1}, \ldots, \psi_{d} \in \mathcal{C}_{q_{1}}^{\infty}(\mathbb{R})$,

$$
\left|E\left[\prod_{j=1}^{d} \psi_{j}\left(F_{j}\right)\right]-\prod_{j=1}^{d} E\left[\psi_{j}\left(F_{j}\right)\right]\right| \leqslant c\left\|\psi_{d}^{\prime}\right\|_{\infty} \prod_{j=1}^{d-1}\left\|\psi_{j}\right\|_{q_{1}} \sum_{1 \leqslant j<\ell \leqslant d} \operatorname{Cov}\left(F_{j}^{2}, F_{\ell}^{2}\right) .
$$

When applied to sequences of multiple stochastic integrals, Theorem 1.2 allows one to deduce the following strong generalization of [9, Theorem 3.4].

Theorem 1.3. Let $d \geqslant 2$ and let $q_{1} \geqslant q_{2} \geqslant \cdots \geqslant q_{d} \geqslant 1$ be fixed integers. For every $n \geqslant 1$, let $F_{n}=\left(F_{1, n}, \ldots, F_{d, n}\right)$ be a d-dimensional random vector such that $F_{j, n}=I_{q_{j}}\left(f_{j, n}\right)$, with $f_{j, n} \in \mathfrak{H}^{\odot q_{j}}$ and $E\left[F_{j, n}^{2}\right]=1$ for all $1 \leqslant j \leqslant d$ and $n \geqslant 1$. Then, the following three conditions are equivalent, as $n \rightarrow \infty$ :

(1) $\operatorname{Cov}\left(F_{i, n}^{2}, F_{j, n}^{2}\right) \rightarrow 0$ for every $1 \leqslant i \neq j \leqslant d$;

(2) $\left\|f_{i, n} \otimes_{r} f_{j, n}\right\| \rightarrow 0$ for every $1 \leqslant i \neq j \leqslant d$ and $1 \leqslant r \leqslant q_{i} \wedge q_{j}$; 
(3) the random variables $F_{1, n}, \ldots, F_{d, n}$ are asymptotically independent, that is, for every collection of smooth test functions $\psi_{1}, \ldots, \psi_{d} \in \mathcal{C}_{q_{1}}^{\infty}(\mathbb{R})$,

$$
E\left[\prod_{j=1}^{d} \psi_{j}\left(F_{j, n}\right)\right]-\prod_{j=1}^{d} E\left[\psi_{j}\left(F_{j, n}\right)\right] \longrightarrow 0 .
$$

We can now state the announced extension of Theorem 1.1 (see Section 1), in which the determinacy condition for the limit random variables $U_{j}$ has been eventually removed.

Theorem 1.4. Let $d \geqslant 2$ and let $q_{1} \geqslant q_{2} \geqslant \cdots \geqslant q_{d} \geqslant 1$ be fixed integers. For every $n \geqslant 1$, let $F_{n}=\left(F_{1, n}, \ldots, F_{d, n}\right)$ be a d-dimensional random vector such that $F_{j, n}=I_{q_{j}}\left(f_{j, n}\right)$, with $f_{j, n} \in \mathfrak{H}^{\odot q_{j}}$ and $E\left[F_{j, n}^{2}\right]=1$ for all $1 \leqslant j \leqslant d$ and $n \geqslant 1$. Let $U_{1}, \ldots, U_{d}$ be independent random variables such that $F_{j, n} \stackrel{\text { law }}{\rightarrow} U_{j}$ as $n \rightarrow \infty$ for every $1 \leqslant j \leqslant d$. Assume that either Condition (1) or Condition (2) of Theorem 1.3 holds. Then, as $n \rightarrow \infty$,

$$
F_{n} \stackrel{\text { law }}{\rightarrow}\left(U_{1}, \ldots, U_{d}\right)
$$

By considering linear combinations, one can also prove the following straightforward generalisations of Theorem 1.3 and Theorem 1.4 (which are potentially useful for applications), where each component of the vector $F_{n}$ is replaced by a multidimensional object. The simple proofs are left to the reader.

Proposition 1.5. Let $d \geqslant 2$, let $q_{1} \geqslant q_{2} \geqslant \cdots \geqslant q_{d} \geqslant 1$ and $m_{1}, \ldots, m_{d} \geqslant 1$ be fixed integers, and set $M:=\sum_{j=1}^{d} m_{j}$. For every $j=1, \ldots, d$, let

$$
\mathbf{F}_{j, n}=\left(F_{j, n}^{(1)}, \ldots, F_{j, n}^{\left(m_{j}\right)}\right):=\left(I_{q_{j}}\left(f_{j, n}^{(1)}\right), \ldots, I_{q_{j}}\left(f_{j, n}^{\left(m_{j}\right)}\right)\right),
$$

where, for $\ell=1, \ldots, m_{j}, f_{j, n}^{(\ell)} \in \mathfrak{H}^{\odot q_{j}}$ and $E\left[\left(F_{j, n}^{(\ell)}\right)^{2}\right]=q_{j} !\left\|f_{j, n}^{(\ell)}\right\|_{\mathfrak{H}^{\otimes q_{j}}}^{2}=1$. Finally, for every $n \geqslant 1$, write $\mathbf{F}_{n}$ to indicate the $M$-dimensional vector $\left(\mathbf{F}_{1, n}, \ldots, \mathbf{F}_{d, n}\right)$. Then, the following three conditions are equivalent, as $n \rightarrow \infty$ :

(1) $\operatorname{Cov}\left(\left(F_{i, n}^{(\ell)}\right)^{2},\left(F_{j, n}^{\left(\ell^{\prime}\right)}\right)^{2}\right) \rightarrow 0$ for every $1 \leqslant i \neq j \leqslant d$, every $\ell=1, \ldots, m_{i}$ and every $\ell^{\prime}=1, \ldots, m_{j}$;

(2) $\left\|f_{i, n}^{(\ell)} \otimes_{r} f_{j, n}^{\left(\ell^{\prime}\right)}\right\| \rightarrow 0$ for every $1 \leqslant i \neq j \leqslant d$, for every $1 \leqslant r \leqslant q_{i} \wedge q_{j}$, every $\ell=1, \ldots, m_{i}$ and every $\ell^{\prime}=1, \ldots, m_{j}$;

(3) the random vectors $\mathbf{F}_{1, n}, \ldots, \mathbf{F}_{d, n}$ are asymptotically independent, that is, for every collection of smooth bounded test functions $\psi_{j} \in \mathcal{C}_{q_{1}}^{\infty}\left(\mathbb{R}^{m_{j}}\right), j=$ $1, \ldots, d$,

$$
E\left[\prod_{j=1}^{d} \psi_{j}\left(\mathbf{F}_{j, n}\right)\right]-\prod_{j=1}^{d} E\left[\psi_{j}\left(\mathbf{F}_{j, n}\right)\right] \longrightarrow 0 .
$$

Proposition 1.6. Let the notation and assumptions of Proposition 1.5 prevail, and assume that either Condition (1) or Condition (2) therein is satisfied. Consider a collection $\left(\mathbf{U}_{1}, \ldots, \mathbf{U}_{d}\right)$ of independent random vectors such that, for $j=1, \ldots, d$, $\mathbf{U}_{j}$ has dimension $m_{j}$. Then, if $\mathbf{F}_{j, n}$ converges in distribution to $\mathbf{U}_{j}$, as $n \rightarrow \infty$, one has also that

$$
\mathbf{F}_{n} \stackrel{\text { law }}{\rightarrow}\left(\mathbf{U}_{1}, \ldots, \mathbf{U}_{d}\right) .
$$


The plan of the paper is as follows. Section 2 contains some further preliminaries related to Gaussian analysis and Malliavin calculus. The proofs of our main results are gathered in Section 3.

\section{Further notation AND Results From Malliavin CALCUlus}

Let $\left\{e_{k}, k \geqslant 1\right\}$ be a complete orthonormal system in $\mathfrak{H}$. Given $f \in \mathfrak{H}^{\odot p}, g \in \mathfrak{H}^{\odot q}$ and $r \in\{0, \ldots, p \wedge q\}$, the $r$ th contraction of $f$ and $g$ is the element of $\mathfrak{H}^{\otimes(p+q-2 r)}$ defined by

$$
f \otimes_{r} g=\sum_{i_{1}, \ldots, i_{r}=1}^{\infty}\left\langle f, e_{i_{1}} \otimes \ldots \otimes e_{i_{r}}\right\rangle_{\mathfrak{H} \otimes r} \otimes\left\langle g, e_{i_{1}} \otimes \ldots \otimes e_{i_{r}}\right\rangle_{\mathfrak{H} \otimes r}
$$

Notice that $f \otimes_{r} g$ is not necessarily symmetric, unless $p=q=r$. We denote its symmetrization by $f \widetilde{\otimes}_{r} g \in \mathfrak{H} \odot(p+q-2 r)$. Moreover, $f \otimes_{0} g=f \otimes g$ equals the tensor product of $f$ and $g$ while, for $p=q, f \otimes_{q} g=\langle f, g\rangle_{\mathfrak{H} \otimes q}$. In the particular case $\mathfrak{H}=$ $L^{2}(A, \mathcal{A}, \mu)$, where $(A, \mathcal{A})$ is a measurable space and $\mu$ is a $\sigma$-finite and non-atomic measure, one has that $\mathfrak{H}^{\odot q}$ can be identified with the space $L_{s}^{2}\left(A^{q}, \mathcal{A}^{\otimes q}, \mu^{\otimes q}\right)$ of $\mu^{q}$-almost everywhere symmetric and square-integrable functions on $A^{q}$. Moreover, for every $f \in \mathfrak{H}^{\odot q}, I_{q}(f)$ coincides with the multiple Wiener-Itô integral of order $q$ of $f$ with respect to $X$ and (2.4) can be written as

$$
\begin{aligned}
\left(f \otimes_{r} g\right)\left(t_{1}, \ldots, t_{p+q-2 r}\right) & =\int_{A^{r}} f\left(t_{1}, \ldots, t_{p-r}, s_{1}, \ldots, s_{r}\right) \\
& \times g\left(t_{p-r+1}, \ldots, t_{p+q-2 r}, s_{1}, \ldots, s_{r}\right) d \mu\left(s_{1}\right) \ldots d \mu\left(s_{r}\right) .
\end{aligned}
$$

We will now introduce some basic elements of the Malliavin calculus with respect to the isonormal Gaussian process $X$ (see again [7,10] for any unexplained notion or result). Let $\mathcal{S}$ be the set of all smooth and cylindrical random variables of the form

$$
F=g\left(X\left(\phi_{1}\right), \ldots, X\left(\phi_{n}\right)\right),
$$

where $n \geqslant 1, g: \mathbb{R}^{n} \rightarrow \mathbb{R}$ is an infinitely differentiable function with compact support, and $\phi_{i} \in \mathfrak{H}$. The Malliavin derivative of $F$ with respect to $X$ is the element of $L^{2}(\Omega, \mathfrak{H})$ defined as

$$
D F=\sum_{i=1}^{n} \frac{\partial g}{\partial x_{i}}\left(X\left(\phi_{1}\right), \ldots, X\left(\phi_{n}\right)\right) \phi_{i} .
$$

By iteration, one can define the $q$ th derivative $D^{q} F$ for every $q \geqslant 2$, which is an element of $L^{2}\left(\Omega, \mathfrak{H}^{\odot q}\right)$.

For $q \geqslant 1$ and $p \geqslant 1, \mathbb{D}^{q, p}$ denotes the closure of $\mathcal{S}$ with respect to the norm $\|\cdot\|_{\mathbb{D}^{q, p}}$, defined by the relation

$$
\|F\|_{\mathbb{D}^{q, p}}^{p}=E\left[|F|^{p}\right]+\sum_{i=1}^{q} E\left[\left\|D^{i} F\right\|_{\mathfrak{H}^{\otimes i}}^{p}\right] .
$$

The Malliavin derivative $D$ verifies the following chain rule. If $\varphi: \mathbb{R}^{n} \rightarrow \mathbb{R}$ is continuously differentiable with bounded partial derivatives and if $F=\left(F_{1}, \ldots, F_{n}\right)$ is a vector of elements of $\mathbb{D}^{1,2}$, then $\varphi(F) \in \mathbb{D}^{1,2}$ and

$$
D \varphi(F)=\sum_{i=1}^{n} \frac{\partial \varphi}{\partial x_{i}}(F) D F_{i} .
$$


Note also that a random variable $F$ as in $(1.2)$ is in $\mathbb{D}^{1,2}$ if and only if

$$
\sum_{q=1}^{\infty} q q !\left\|f_{q}\right\|_{\mathfrak{H}^{\otimes q}}^{2}<\infty
$$

and, in this case, $E\left[\|D F\|_{\mathfrak{H}}^{2}\right]=\sum_{q \geqslant 1} q q !\left\|f_{q}\right\|_{\mathfrak{H} \otimes q}^{2}$. If $\mathfrak{H}=L^{2}(A, \mathcal{A}, \mu)$ (with $\mu$ nonatomic), then the derivative of a random variable $F$ as in (1.2) can be identified with the element of $L^{2}(A \times \Omega)$ given by

$$
D_{a} F=\sum_{q=1}^{\infty} q I_{q-1}\left(f_{q}(\cdot, a)\right), \quad a \in A .
$$

We denote by $\delta$ the adjoint of the operator $D$, also called the divergence operator. A random element $u \in L^{2}(\Omega, \mathfrak{H})$ belongs to the domain of $\delta$, noted $\operatorname{Dom} \delta$, if and only if it verifies

$$
\left|E\left[\langle D F, u\rangle_{\mathfrak{H}}\right]\right| \leqslant c_{u} \sqrt{E\left[F^{2}\right]}
$$

for any $F \in \mathbb{D}^{1,2}$, where $c_{u}$ is a constant depending only on $u$. If $u \in \operatorname{Dom} \delta$, then the random variable $\delta(u)$ is defined by the duality relationship (customarily called 'integration by parts formula'):

$$
E[F \delta(u)]=E\left[\langle D F, u\rangle_{\mathfrak{H}}\right]
$$

which holds for every $F \in \mathbb{D}^{1,2}$. The formula (2.8) extends to the multiple Skorohod integral $\delta^{q}$, and we have

$$
E\left[F \delta^{q}(u)\right]=E\left[\left\langle D^{q} F, u\right\rangle_{\mathfrak{H}^{\otimes q}}\right]
$$

for any element $u$ in the domain of $\delta^{q}$ and any random variable $F \in \mathbb{D}^{q, 2}$. Moreover, $\delta^{q}(h)=I_{q}(h)$ for any $h \in \mathfrak{H}^{\odot q}$.

The following property, corresponding to [6, Lemma 2.1], will be used in the paper. Let $q \geqslant 1$ be an integer, suppose that $F \in \mathbb{D}^{q, 2}$, and let $u$ be a symmetric element in $\operatorname{Dom} \delta^{q}$. Assume that $\left\langle D^{r} F, \delta^{j}(u)\right\rangle_{\mathfrak{H}^{\otimes r}} \in L^{2}\left(\Omega, \mathfrak{H}^{\otimes q-r-j}\right)$ for any $0 \leqslant$ $r+j \leqslant q$. Then $\left\langle D^{r} F, u\right\rangle_{\mathfrak{H} \otimes r}$ belongs to the domain of $\delta^{q-r}$ for any $r=0, \ldots, q-1$, and we have

$$
F \delta^{q}(u)=\sum_{r=0}^{q}\left(\begin{array}{l}
q \\
r
\end{array}\right) \delta^{q-r}\left(\left\langle D^{r} F, u\right\rangle_{\mathfrak{H}^{\otimes r}}\right) .
$$

(We use the convention that $\delta^{0}(v)=v, v \in \mathbb{R}$, and $D^{0} F=F, F \in L^{2}(\Omega)$.)

For any Hilbert space $V$, we denote by $\mathbb{D}^{k, p}(V)$ the corresponding Sobolev space of $V$-valued random variables (see [10, page 31]). The operator $\delta^{q}$ is continuous from $\mathbb{D}^{k, p}\left(\mathfrak{H}^{\otimes q}\right)$ to $\mathbb{D}^{k-q, p}$, for any $p>1$ and any integers $k \geq q \geq 1$, and one has the estimate

$$
\left\|\delta^{q}(u)\right\|_{\mathbb{D}^{k-q, p}} \leqslant c_{k, p}\|u\|_{\mathbb{D}^{k, p}(\mathfrak{H} \otimes q)}
$$

for all $u \in \mathbb{D}^{k, p}\left(\mathfrak{H}^{\otimes q}\right)$, and some constant $c_{k, p}>0$. These inequalities are direct consequences of the so-called Meyer inequalities (see [10, Proposition 1.5.7] for the case $q=1$, and in the case $q>1$ we can use an iterative argument). In particular, these estimates imply that $\mathbb{D}^{q, 2}\left(\mathfrak{H}^{\otimes q}\right) \subset$ Dom $\delta^{q}$ for any integer $q \geq 1$.

The operator $L$ is defined on the Wiener chaos expansion as

$$
L=\sum_{q=0}^{\infty}-q J_{q}
$$


and is called the infinitesimal generator of the Ornstein-Uhlenbeck semigroup. The domain of this operator in $L^{2}(\Omega)$ is the set

$$
\operatorname{Dom} L=\left\{F \in L^{2}(\Omega): \sum_{q=1}^{\infty} q^{2}\left\|J_{q} F\right\|_{L^{2}(\Omega)}^{2}<\infty\right\}=\mathbb{D}^{2,2} .
$$

There is an important relationship between the operators $D, \delta$ and $L$. A random variable $F$ belongs to the domain of $L$ if and only if $F \in \operatorname{Dom}(\delta D)$ (i.e. $F \in \mathbb{D}^{1,2}$ and $D F \in \operatorname{Dom} \delta$ ), and in this case

$$
\delta D F=-L F .
$$

We also define the operator $L^{-1}$, which is the pseudo-inverse of $L$, as follows: for every $F \in L^{2}(\Omega)$ with zero mean, we set $L^{-1} F=\sum_{q \geqslant 1}-\frac{1}{q} J_{q}(F)$. We note that $L^{-1}$ is an operator with values in $\mathbb{D}^{2,2}$ and that $L L^{-1} F=E-E[F]$ for any $F \in L^{2}(\Omega)$.

\section{Proofs of the Results stated in SeCtion 1.3}

3.1. Proof of Theorem 1.2. The proof of Theorem 1.2 is based on a recursive application of the following quantitative result, whose proof has been inspired by the pioneering work of Üstünel and Zakai on the characterization of the independence on Wiener chaos (see [15]).

Proposition 3.1. Let $m \geqslant 1$ and $p_{1}, \ldots, p_{m}, q$ be integers such that $p_{j} \geqslant q$ for every $j=1, \ldots, m$. There exists a constant $c$, uniquely depending on $m$ and $p_{1}, \ldots, p_{m}, q$, such that one has the bound

$$
|E[\varphi(F) \psi(G)]-E[\varphi(F)] E[\psi(G)]| \leq c\left\|\psi^{\prime}\right\|_{\infty}\|\varphi\|_{q} \sum_{j=1}^{m} \operatorname{Cov}\left(F_{j}^{2}, G^{2}\right),
$$

for every vector $F=\left(F_{1}, \ldots, F_{m}\right)$ such that $F_{j}=I_{p_{j}}\left(f_{j}\right), f_{j} \in \mathfrak{H}^{\odot p_{j}}$ and $E\left[F_{j}^{2}\right]=1$ $(j=1, \ldots, m)$, for every random variable $G=I_{q}(g), g \in \mathfrak{H}^{\odot q}$, and for every pair of smooth test functions $\varphi \in \mathcal{C}_{q}^{\infty}\left(\mathbb{R}^{m}\right)$ and $\psi \in \mathcal{C}_{1}^{\infty}(\mathbb{R})$.

Proof. Using the chain rule (2.6) together with the relation $-D L^{-1}=(I-L)^{-1} D$ (see, e.g., [12]), one has

$$
\varphi(F)-E[\varphi(F)]=L L^{-1} \varphi(F)=-\delta\left(D L^{-1} \varphi(F)\right)=\sum_{j=1}^{m} \delta\left((I-L)^{-1} \partial_{j} \varphi(F) D F_{j}\right),
$$

from which, by using (2.8), one deduces that

$$
\begin{aligned}
& E[\varphi(F) \psi(G)]-E[\varphi(F)] E[\psi(G)]=\sum_{j=1}^{m} E\left[\left\langle(I-L)^{-1} \partial_{j} \varphi(F) D F_{j}, D G\right\rangle_{\mathfrak{H}} \psi^{\prime}(G)\right] \\
\leq & \left\|\psi^{\prime}\right\|_{\infty} \sum_{j=1}^{m} E\left[\left|\left\langle(I-L)^{-1} \partial_{j} \varphi(F) D F_{j}, D G\right\rangle_{\mathfrak{H}}\right|\right] .
\end{aligned}
$$

Then, the result follows by applying the next lemma to each term in the previous sum and taking $\phi=\partial_{j} \varphi$, for $j=1, \ldots, m$.

Lemma 3.2. Under the assumptions of Proposition 3.1, for any smooth function $\phi \in \mathcal{C}_{q-1}^{\infty}\left(\mathbb{R}^{m}\right)$ and for any $j=1, \ldots, m$, we have

$$
E\left|\left\langle(I-L)^{-1} \phi(F) D F_{j}, D G\right\rangle_{\mathfrak{H}}\right| \leq c\|\phi\|_{q-1} \operatorname{Cov}\left(F^{2}, G^{2}\right) .
$$


Proof. Throughout the proof, the symbol $c$ will denote a positive finite constant uniquely depending on $m$ and $p_{1}, \ldots, p_{m}, q$, whose value may change from line to line. As it is standard, without loss of generality, we can assume that the underlying Hilbert space $\mathfrak{H}$ is of the form $L^{2}(A, \mathcal{A}, \mu)$, where $\mu$ is a $\sigma$-finite measure without atoms. It follows that

$\left\langle(I-L)^{-1} \phi(F) D F_{j}, D G\right\rangle_{\mathfrak{H}}=p_{j} q \int_{A}\left[(I-L)^{-1} \phi(F) I_{p_{j}-1}\left(f_{j}(\cdot, \theta)\right)\right] I_{q-1}(g(\cdot, \theta)) \mu(d \theta)$.

Now we apply the formula (2.10) to $u=g(\cdot, \theta)$ and $F=(I-L)^{-1} \phi(F) I_{p_{j}-1}\left(f_{j}(\cdot, \theta)\right)$ and we obtain, using $D^{r}(I-L)^{-1}=((r+1) I-L)^{-1} D^{r}$ as well (see, e.g., [12]),

$$
\begin{aligned}
& =\sum_{r=0}^{q-1}\left(\begin{array}{c}
q-1 \\
r
\end{array}\right) \delta^{q-1-r}\left(\left\langle g(\cdot, \theta), D^{r}\left[(I-L)^{-1} \phi(F) I_{p_{j}-1}\left(f_{j}(\cdot, \theta)\right)\right]\right\rangle_{\mathfrak{H} \otimes r}\right) \\
& =\sum_{r=0}^{q-1}\left(\begin{array}{c}
q-1 \\
r
\end{array}\right) \delta^{q-1-r}\left(\left\langle g(\cdot, \theta),((r+1) I-L)^{-1} D^{r}\left[\phi(F) I_{p_{j}-1}\left(f_{j}(\cdot, \theta)\right)\right]\right\rangle_{\mathfrak{H} \otimes r}\right) .
\end{aligned}
$$

Now, substituting (3.15) into (3.14) yields

$$
\begin{aligned}
& \left\langle(I-L)^{-1} \phi(F) D F_{j}, D G\right\rangle_{\mathfrak{H}}=p_{j} q \sum_{r=0}^{q-1}\left(\begin{array}{c}
q-1 \\
r
\end{array}\right) \\
& \times \delta^{q-1-r}\left(\int_{A^{r+1}} g\left(\cdot, \mathbf{s}^{r+1}\right)((r+1) I-L)^{-1} D_{s_{1}, \ldots, s_{r}}^{r}\left[\phi(F) I_{p_{j}-1}\left(f_{j}\left(\cdot, s_{r+1}\right)\right)\right] \mu\left(d \mathbf{s}^{r+1}\right)\right),
\end{aligned}
$$

where $\mathbf{s}^{r+1}=\left(s_{1}, \ldots, s_{r+1}\right)$. We have, by the Leibniz rule,

$$
\begin{aligned}
D_{s_{1}, \ldots, s_{r}}^{r} & {\left[\phi(F) I_{p_{j}-1}\left(f_{j}\left(\cdot, s_{r+1}\right)\right)\right] } \\
\quad & =\sum_{\alpha=0}^{r}\left(\begin{array}{l}
r \\
\alpha
\end{array}\right) D_{s_{1}, \ldots, s_{\alpha}}^{\alpha}[\phi(F)] D_{s_{\alpha+1}, \ldots, s_{r}}^{r-\alpha}\left[I_{p_{j}-1}\left(f_{j}\left(\cdot, s_{r+1}\right)\right)\right] \\
& =\sum_{\alpha=0}^{r}\left(\begin{array}{l}
r \\
\alpha
\end{array}\right) \frac{\left(p_{j}-1\right) !}{\left(p_{j}-r+\alpha-1\right) !} D_{s_{1}, \ldots, s_{\alpha}}^{\alpha}[\phi(F)] I_{p_{j}-r+\alpha-1}\left(f_{j}\left(\cdot, s_{\alpha+1}, \ldots, s_{r+1}\right)\right) .
\end{aligned}
$$

Fix $0 \leq r \leq q-1$ and $0 \leq \alpha \leq r$. It suffices to estimate the following expectation:

$$
\begin{aligned}
& E \mid \delta^{q-1-r}\left(\int_{A^{r+1}} g\left(\cdot, \mathbf{s}^{\alpha}, \mathbf{t}^{r-\alpha+1}\right)\right. \\
& \left.\times((r+1) I-L)^{-1} D_{\mathbf{s}^{\alpha}}^{\alpha}[\phi(F)] I_{p_{j}-r+\alpha-1}\left(f_{j}\left(\cdot, \mathbf{t}^{r-\alpha+1}\right)\right) \mu\left(d \mathbf{s}^{\alpha}\right) \mu\left(d \mathbf{t}^{r-\alpha+1}\right)\right) \mid .
\end{aligned}
$$

Note that, in the previous formula, the symbol '. ' inside the argument of the kernel $g$ represents $q-1-r$ variables that are integrated with respect to the multiple Skorohod integral $\delta^{q-1-r}$, whereas the '.' inside the argument of $f_{j}$ stands for $p_{j}-r+\alpha-1$ variables that are integrated with respect to the multiple Wiener-Itô integral $I_{p_{j}-r+\alpha-1}$. By Meyer's inequalities (2.11), we can estimate the expectation 
(3.16), up to a universal constant, by the sum over $0 \leqslant \beta \leqslant q-r-1$ of the quantities

$$
\begin{aligned}
& \left(\int _ { A ^ { q - r - 1 + \beta } } E \left(\int _ { A ^ { r + 1 } } g ( \mathbf { v } ^ { q - r - 1 } , \mathbf { s } ^ { \alpha } , \mathbf { t } ^ { r - \alpha + 1 } ) D _ { \mathbf { u } ^ { \beta } } ^ { \beta } \left\{((r+1) I-L)^{-1} D_{\mathbf{s}^{\alpha}}^{\alpha}[\phi(F)]\right.\right.\right. \\
& \left.\left.\left.\times I_{p_{j}-r+\alpha-1}\left(f_{j}\left(\cdot, \mathbf{t}^{r-\alpha+1}\right)\right)\right\} \mu\left(d \mathbf{s}^{\alpha}\right) \mu\left(d \mathbf{t}^{r-\alpha+1}\right)\right)^{2} \mu\left(d \mathbf{v}^{q-r-1}\right) \mu\left(d \mathbf{u}^{\beta}\right)\right)^{\frac{1}{2}} \\
& =\left(\int _ { A ^ { q - r - 1 + \beta } } E \left(( ( \beta + r + 1 ) I - L ) ^ { - 1 } \int _ { A ^ { r + 1 } } g ( \mathbf { v } ^ { q - r - 1 } , \mathbf { s } ^ { \alpha } , \mathbf { t } ^ { r - \alpha + 1 } ) D _ { \mathbf { u } ^ { \beta } } ^ { \beta } \left\{D_{\mathbf{s}^{\alpha}}^{\alpha}[\phi(F)]\right.\right.\right. \\
& \left.\left.\left.\left.\times I_{p_{j}-r+\alpha-1}\left(f_{j}\left(\cdot, \mathbf{t}^{r-\alpha+1}\right)\right)\right\} \mu\left(d \mathbf{s}^{\alpha}\right) \mu\left(d \mathbf{t}^{r-\alpha+1}\right)\right)\right)^{2} \mu\left(d \mathbf{v}^{q-r-1}\right) \mu\left(d \mathbf{u}^{\beta}\right)\right)^{\frac{1}{2}} \\
& \leq c\left(\int _ { A ^ { q - r - 1 + \beta } } E \left(\int _ { A ^ { r + 1 } } g ( \mathbf { v } ^ { q - r - 1 } , \mathbf { s } ^ { \alpha } , \mathbf { t } ^ { r - \alpha + 1 } ) D _ { \mathbf { u } ^ { \beta } } ^ { \beta } \left\{D_{\mathbf{s}^{\alpha}}^{\alpha}[\phi(F)]\right.\right.\right. \\
& \left.\left.\left.\left.\times I_{p_{j}-r+\alpha-1}\left(f_{j}\left(\cdot, \mathbf{t}^{r-\alpha+1}\right)\right)\right\} \mu\left(d \mathbf{s}^{\alpha}\right) \mu\left(d \mathbf{t}^{r-\alpha+1}\right)\right)\right)^{2} \mu\left(d \mathbf{v}^{q-r-1}\right) \mu\left(d \mathbf{u}^{\beta}\right)\right)^{\frac{1}{2}} .
\end{aligned}
$$

Thanks to the Leibniz formula, the last bound implies that we need to estimate, for any $0 \leq \eta \leq \beta \leq q-r-1$, the following quantity:

$$
\begin{aligned}
& \left(\int _ { A ^ { q - r - 1 + \beta } } E \left(\int_{A^{r+1}} g\left(\mathbf{v}^{q-r-1}, \mathbf{s}^{\alpha}, \mathbf{t}^{r-\alpha+1}\right) D_{\mathbf{s}^{\alpha}, \mathbf{w}^{\eta}}^{\alpha+\eta}[\phi(F)]\right.\right. \\
& \left.\left.\times I_{p_{j}-r+\alpha-1+\eta-\beta}\left(f_{j}\left(\cdot, \mathbf{t}^{r-\alpha+1}, \mathbf{y}^{\beta-\eta}\right)\right) \mu\left(d \mathbf{s}^{\alpha}\right) \mu\left(d \mathbf{t}^{r-\alpha+1}\right)\right)^{2} \mu\left(d \mathbf{v}^{q-r-1}\right) \mu\left(d \mathbf{w}^{\eta}\right) \mu\left(d \mathbf{y}^{\beta-\eta}\right)\right)^{\frac{1}{2}} .
\end{aligned}
$$

We can rewrite this quantity as

$$
\begin{aligned}
& \left(\int_{A^{q-r-1-\beta}} E\left(\left(I_{p_{j}-r+\alpha-1+\eta-\beta}\left(f_{j}\left(\cdot, \mathbf{y}^{\beta-\eta}\right)\right) \otimes_{r-\alpha+1} g\left(\cdot, \mathbf{v}^{q-r-1}\right)\right) \otimes_{\alpha} D^{\alpha+\eta}[\phi(F)]\left(\mathbf{w}^{\eta}\right)\right)^{2}\right. \\
& \left.\times \mu\left(d \mathbf{y}^{\beta-\eta}\right) \mu\left(d \mathbf{w}^{\eta}\right) \mu\left(d \mathbf{v}^{q-r-1}\right)\right)^{\frac{1}{2}} .
\end{aligned}
$$

Applying the Cauchy-Schwarz inequality yields that such a quantity is bounded by

$$
\begin{aligned}
& \left(E\left[\left\|I_{p_{j}-r+\alpha-1+\eta-\beta}\left(f_{j}\right) \otimes_{r-\alpha+1} g\right\|^{2}\left\|D^{\alpha+\eta}[\phi(F)]\right\|^{2}\right]\right)^{\frac{1}{2}} \\
\leq \quad & \left(E\left\|I_{p_{j}-r+\alpha-1+\eta-\beta}\left(f_{j}\right) \otimes_{r-\alpha+1} g\right\|^{4}\right)^{\frac{1}{4}}\left(E\left\|D^{\alpha+\eta}[\phi(F)]\right\|^{4}\right)^{\frac{1}{4}} .
\end{aligned}
$$

Set $\gamma=\alpha+\eta$. Applying the generalised Faá di Bruno's formula (see, e.g., 3]) we deduce that

$$
\begin{aligned}
& D^{\gamma}[\phi(F)] \\
& =\sum \frac{\gamma !}{\prod_{i=1}^{\gamma} i !^{k_{i}} \prod_{i=1}^{\gamma} \prod_{j=1}^{m} q_{i j} !} \frac{\partial^{k} \phi(F)}{\partial x_{1}^{p_{1}} \cdots \partial x_{m}^{p_{m}}} \prod_{i=1}^{\gamma \wedge p^{*}}\left(D^{i} F_{1}\right)^{\otimes q_{i 1}} \otimes \cdots \otimes\left(D^{i} F_{m}\right)^{\otimes q_{i m}}
\end{aligned}
$$


where $p^{*}=\min \left\{p_{1}, \ldots, p_{m}\right\}$, and the sum runs over all non-negative integer solutions of the system of $\gamma+1$ equations

$$
\begin{aligned}
& k_{1}+2 k_{2}+\cdots+k_{\gamma}=\gamma, \\
& q_{11}+q_{12}+\cdots+q_{1 m}=k_{1}, \\
& q_{21}+q_{22}+\cdots+q_{2 m}=k_{2}, \\
& \ldots \ldots \\
& q_{\gamma 1}+q_{\gamma 2}+\cdots+q_{\gamma m}=k_{\gamma},
\end{aligned}
$$

and we have moreover set $p_{j}=q_{1 j}+\cdots+q_{\gamma j}, j=1, \ldots, r$, and $k=p_{1}+\cdots+p_{m}=$ $k_{1}+\cdots+k_{\gamma}$. This expression yields immediately that

$$
\left\|D^{\gamma}[\phi(F)]\right\| \leq c\|\phi\|_{q-1} \sum \prod_{i=1}^{\gamma \wedge p^{*}}\left\|D^{i} F_{1}\right\|^{q_{i 1}} \cdots\left\|D^{i} F_{m}\right\|^{q_{i m}}
$$

and using the facts that all $\mathbb{D}^{k, p}$ norms $(k, p \geqslant 1)$ are equivalent on a fixed Wiener chaos and that the elements of the vector $F$ have unit variance by assumption, we infer that

$$
\left(E\left\|D^{\gamma}[\phi(F)]\right\|^{4}\right)^{\frac{1}{4}} \leq c\|\phi\|_{q-1} .
$$

On the other hand, using that all the $L^{p}$-norms, $p \geq 2$, are equivalent on a fixed Wiener chaos, as a consequence of the hypercontractivity property of the OrnsteinUhlenbeck semigroup (see, e.g., [7, Corollary 2.8.14]), one has that

$$
\left(E\left\|I_{p_{j}-r+\alpha-1+\eta-\beta}\left(f_{j}\right) \otimes_{r-\alpha+1} g\right\|^{4}\right)^{\frac{1}{4}} \leq c\left(E\left\|I_{p_{j}-r+\alpha-1+\eta-\beta}\left(f_{j}\right) \otimes_{r-\alpha+1} g\right\|^{2}\right)^{\frac{1}{2}} .
$$

Since

$$
E\left\|I_{p_{j}-r+\alpha-1+\eta-\beta}\left(f_{j}\right) \otimes_{r-\alpha+1} g\right\|^{2}=\left(p_{j}-r+\alpha-1+\eta-\beta\right) !\left\|f_{j} \otimes_{r-\alpha+1} g\right\|^{2},
$$

and

$$
\max _{1 \leq r \leq q}\left\|f_{j} \otimes_{r} g\right\| \leqslant \operatorname{Cov}\left(F_{j}^{2}, G^{2}\right) \quad \text { (see, e.g., [9, inequality (3.26)]), }
$$

we finally obtain the estimate (3.13), thus concluding the proof.

Proof of Theorem 1.2. Just observe that

$$
\begin{aligned}
&\left|E\left[\prod_{j=1}^{d} \psi_{j}\left(F_{j}\right)\right]-\prod_{j=1}^{d} E\left[\psi_{j}\left(F_{j}\right)\right]\right| \\
& \leqslant \sum_{j=2}^{d} \mid E\left[\psi_{1}\left(F_{1}\right) \cdots \psi_{j-1}\left(F_{j-1}\right)\right] E\left[\psi_{j}\left(F_{j}\right)\right] \cdots E\left[\psi_{d}\left(F_{d}\right)\right] \\
&-E\left[\psi_{1}\left(F_{1}\right) \cdots \psi_{j}\left(F_{j}\right)\right] E\left[\psi_{j+1}\left(F_{j+1}\right)\right] \cdots E\left[\psi_{d}\left(F_{d}\right)\right] \mid,
\end{aligned}
$$

so that the conclusion is achieved (after some routine computations) by applying Proposition 3.1 (in the case $m=j-1, p_{i}=q_{i}, i=1, \ldots, j-1$, and $q=q_{j}$ ) to each summand on the right-hand side of the previous estimate. 
3.2. Proof of Theorem 1.3. The equivalence between (1) and (2) follows from [9. Theorem 3.4]. That (3) implies (1) would have been immediate if the square function $x \mapsto x^{2}$ were bounded. To overcome this slight difficulty, it suffices to combine the hypercontractivity property of chaotic random variables (from which it follows that our sequence $\left(F_{n}\right)$ is bounded in $L^{p}(\Omega)$ for any $p \geqslant 1$ ) with a standard approximation argument. Finally, the implication $(1) \Rightarrow(3)$ is a direct consequence of (1.3).

3.3. Proof of Theorem 1.4. Assume that there exists a subsequence of $\left\{F_{n}\right\}$ converging in distribution to some limit $\left(V_{1}, \ldots, V_{d}\right)$. For any collection of smooth test functions $\psi_{1}, \ldots, \psi_{d}: \mathbb{R} \rightarrow \mathbb{R}$, one can then write

$$
E\left[\prod_{j=1}^{d} \psi_{j}\left(V_{j}\right)\right]=\prod_{j=1}^{d} E\left[\psi_{j}\left(V_{j}\right)\right]=\prod_{j=1}^{d} E\left[\psi_{j}\left(U_{j}\right)\right]=E\left[\prod_{j=1}^{d} \psi_{j}\left(U_{j}\right)\right] .
$$

Indeed, the first equality in (3.17) is a direct consequence of the point of Theorem 1.3. the second one follows from the fact that $V_{j} \stackrel{\text { law }}{=} U_{j}$ for any $j$ by assumption, and the last one follows from the independence of the $U_{j}$. Thus, we deduce from (3.17) that $\left(U_{1}, \ldots, U_{d}\right)$ is the only possible limit in law for any converging subsequence extracted from $\left\{F_{n}\right\}$. Since the sequence $\left\{F_{n}\right\}$ is tight (indeed, it is bounded in $\left.L^{2}(\Omega)\right)$, one deduces that $F_{n} \stackrel{\text { law }}{\rightarrow}\left(U_{1}, \ldots, U_{d}\right)$, which completes the proof of Theorem 1.4 .

\section{ACKNOWLEDGMENT}

We would like to thank the referee for the careful reading and for a list of comments and suggestions.

\section{REFERENCES}

[1] Shuyang Bai and Murad S. Taqqu, Multivariate limit theorems in the context of longrange dependence, J. Time Series Anal. 34 (2013), no. 6, 717-743, DOI 10.1111/jtsa.12046. MR3127215

[2] Solesne Bourguin and Jean-Christophe Breton, Asymptotic Cramér type decomposition for Wiener and Wigner integrals, Infin. Dimens. Anal. Quantum Probab. Relat. Top. 16 (2013), no. 1, 1350005, 13, DOI 10.1142/S0219025713500057. MR3071457

[3] Rumen L. Mishkov, Generalization of the formula of Faa di Bruno for a composite function with a vector argument, Int. J. Math. Math. Sci. 24 (2000), no. 7, 481-491, DOI 10.1155/S0161171200002970. MR1781515 (2001f:26016)

[4] Ivan Nourdin, Lectures on Gaussian approximations with Malliavin calculus, Séminaire de Probabilités XLV, Lecture Notes in Math., vol. 2078, Springer, Cham, 2013, pp. 3-89, DOI 10.1007/978-3-319-00321-4_1. MR3185909

[5] Ivan Nourdin, A webpage on Stein's method and Malliavin calculus, https://sites.google. $\mathrm{com} / \mathrm{site} / \mathrm{malliavinstein}$

[6] Ivan Nourdin and David Nualart, Central limit theorems for multiple Skorokhod integrals, J. Theoret. Probab. 23 (2010), no. 1, 39-64, DOI 10.1007/s10959-009-0258-y. MR.2591903 (2011d:60075)

[7] Ivan Nourdin and Giovanni Peccati, Normal approximations with Malliavin calculus, Cambridge Tracts in Mathematics, vol. 192, Cambridge University Press, Cambridge, 2012. From Stein's method to universality. MR2962301

[8] Ivan Nourdin and Giovanni Peccati (2013): The optimal fourth moment theorem. To appear in: Proceedings of the American Mathematical Society. 
[9] Ivan Nourdin and Jan Rosiński, Asymptotic independence of multiple Wiener-Itô integrals and the resulting limit laws, Ann. Probab. 42 (2014), no. 2, 497-526, DOI 10.1214/12AOP826. MR 3178465

[10] David Nualart, The Malliavin calculus and related topics, 2nd ed., Probability and its Applications (New York), Springer-Verlag, Berlin, 2006. MR2200233 (2006j:60004)

[11] David Nualart and Giovanni Peccati, Central limit theorems for sequences of multiple stochastic integrals, Ann. Probab. 33 (2005), no. 1, 177-193, DOI 10.1214/009117904000000621. MR2118863(2005k:60077)

[12] David Nualart and Moshe Zakai, A summary of some identities of the Malliavin calculus, Stochastic partial differential equations and applications, II (Trento, 1988), Lecture Notes in Math., vol. 1390, Springer, Berlin, 1989, pp. 192-196, DOI 10.1007/BFb0083946. MR 1019603 (91d:60129)

[13] Giovanni Peccati and Ciprian A. Tudor, Gaussian limits for vector-valued multiple stochastic integrals, Séminaire de Probabilités XXXVIII, Lecture Notes in Math., vol. 1857, Springer, Berlin, 2005, pp. 247-262. MR2126978 (2006i:60071)

[14] Eric V. Slud, The moment problem for polynomial forms in normal random variables, Ann. Probab. 21 (1993), no. 4, 2200-2214. MR.1245307 (95c:60019)

[15] Ali Süleyman Üstünel and Moshe Zakai, On independence and conditioning on Wiener space, Ann. Probab. 17 (1989), no. 4, 1441-1453. MR1048936 (91g:60068)

Université du Luxembourg, Unité de Recherche en Mathématiques, 6 Rue Richard Coudenhove-Kalergi, L-1359, LuxembourG

E-mail address: ivan.nourdin@uni.lu

Department of Mathematics, University of Kansas, Lawrence, Kansas 66045

E-mail address: nualart@math.ku.edu

Université du Luxembourg, Unité de Recherche en Mathématiques, 6 Rue Richard Coudenhove-Kalergi, L-1359, Luxembourg

E-mail address: giovanni.peccati@gmail.com 